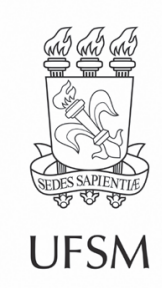

\title{
Artigos
}

\section{Glyphosate e imazapyr no controle de brotações de eucalipto: aplicação no fuste principal anterior ao corte da árvore}

\author{
Glyphosate and imazapyr to eucalyptus sprout control: application to the \\ main stem before the harvesting of the tree
}

\section{William Gomes Montes ${ }^{\oplus}$, Leonardo David Tuffi Santos" $\oplus^{\bullet}$, Rodrigo Eduardo Barros" ${ }^{\prime \prime}$, Wellington Almeida"' ${ }^{\oplus}$, Antônio Junior Silva Abreu"l' ${ }^{\circ}$, Matheus Mendes Reisiv ${ }^{\oplus}$, Luan Mateus Silva Donato" ${ }^{\bullet}$, Guilherme Augusto de Paiva Ferreira" $\oplus^{\circ}$}

\author{
'Celulose Nipo-Brasileira S.A., Belo Oriente, MG, Brasil \\ "Universidade Federal de Minas Gerais, Montes Claros, MG, Brasil \\ '"Gelf Siderurgia S.A., Itacambira, MG, Brasil \\ IVInstituto Federal do Norte de Minas Gerais, Araçuaí, MG, Brasil
}

\section{RESUMO}

A pulverização de herbicidas sobre a rebrota do eucalipto é uma prática comum no setor florestal. Entretanto, observa-se a necessidade de várias operações em uma mesma área para controle das brotações. Objetivou-se avaliar a eficiência dos herbicidas glyphosate e imazapyr, aplicados no fuste principal antes do corte raso das árvores, no controle de brotações de eucalipto. Foram implantados sete tratamentos representados por doses de 3,0;4,0 e 5,0 mL/fuste de glyphosate (Gli-Up ${ }^{\circledR} 360 \mathrm{~g}$ i.a $\mathrm{L}^{-1}$ ) e das doses de 0,3; 0,5 e 0,7 mL/fuste de imazapyr (Chopper Florestal ${ }^{\circledR} 250$ g i.a. $\mathrm{L}^{-1}$ ), além de um tratamento-testemunha. A aplicação dos herbicidas foi realizada em inserções feitas nos fustes das árvores. Aos 30 dias após aplicação dos herbicidas, realizou-se o corte das árvores. Aos 150 dias após o corte das árvores, foram realizadas avaliações do número de brotações por cepa, número de cepas com presença de brotações, altura e a eficiência de controle das brotações. A aplicação de glyphosate promoveu redução do vigor das brotações. Porém, observou-se emissão de brotações em pelo menos $80 \%$ das cepas avaliadas. A aplicação da dose de $3 \mathrm{~mL}$ de glyphosate promoveu porcentagens de $80 \%$ de eficiência de controle. Contudo, observou-se que operações de controle adicionais seriam necessárias. A aplicação de imazapyr mostrou-se ineficiente com controle inferior a $25 \%$. Portanto, conclui-se que a aplicação de glyphosate no fuste da árvore em pré-corte reduz o vigor de brotações de eucalipto. Adicionalmente, a aplicação de imazapyr, nas doses e forma de aplicação testadas, não é recomendada para controle de brotações de eucalipto.

Palavras-chave: Eucalyptus grandis x Eucalyptus urophylla; Erradicação de brotações; Brotações indesejadas; Controle químico 


\section{ABSTRACT}

Herbicide spraying on eucalyptus regrowth prior to planting reform is a common practice in the forest sector. However, several operations are needed in the same area for an effective sprout control. The objective of this work was to evaluate the efficiency of the herbicides glyphosate and imazapyr, applied to the main stem before the harvesting of the tree to eucalyptus sprouts control. There were set up 7 treatments, with application of doses of 3.0; 4.0; and $5.0 \mathrm{~mL} / \mathrm{stem}$ of glyphosate (Gli-Up ${ }^{\circledR} 360 \mathrm{~g} \mathrm{i.a} \mathrm{L-1}^{-1}$ ) and doses of 0.3; 0.5; and $0.7 \mathrm{~mL} / \mathrm{stem}$ of imazapyr (Chopper Florestal ${ }^{\circledR} 250 \mathrm{~g}$ i.a. $\mathrm{L}^{-1}$ ), in addition to a control treatment. The herbicide spraying consisted of the directed spraying of the herbicides on incisions carried out on the stem of the trees. The harvest of the trees was carried out 30 days after the herbicide spraying. Assessments about the number of sprouts per stump were carried out, the number of stump with presence of coppice, height and control efficiency of the coppice 150 days after harvesting. The glyphosate spraying promoted reduction of the sprout development and vigor. However, even with this reduction, it was observed presence of sprouts in at least $80 \%$ of the assessed stumps. The spraying of the dose of $3 \mathrm{~mL}$ of glyphosate promoted values next to $80 \%$ of control efficiency. Nevertheless, it was observed that new control operations would be necessary. The spraying of imazapyr was inefficient with control efficiency values less than $25 \%$. In conclusion, the application of glyphosate to the main stem before the harvesting of the tree presents reduction in the eucalyptus sprout development. Additionally, the application of imazapyr, in the doses and form application tested, is not recommended to eucalyptus sprout control.

Keywords: Eucalyptus grandis x Eucalyptus urophylla; Sprouts eradication; Unwanted sprouts; Chemistry control

\section{INTRODUÇÃO}

As florestas plantadas representam aproximadamente 7\% da área de florestas em termos mundiais (VILLAR et al., 2011; PAYN et al., 2015), mas, ainda assim, auxiliam no suprimento da crescente demanda de madeira no mundo e concomitantemente reduzem a pressão sobre florestas nativas (BAUHUS; VAN DER MEER; KANNINEN, 2010; VIDAL et al., 2015). O Brasil se destaca no cenário mundial por apresentar cultivos com elevada produtividade (PAYN et al., 2015) caracterizados por altas densidades de plantio e baixos períodos de rotação (FERRAZ FILHO; SCOLFORO; MOLA-YUDEGO, 2014).

No entanto, para obtenção de elevadas taxas de produtividade são necessárias práticas silviculturais adequadas, principalmente no estabelecimento do plantio. Entre essas práticas, destaca-se o manejo de plantas daninhas com objetivo de minimizar 
seus efeitos competitivos com mudas implantadas na reforma dos plantios. Porém, além das plantas daninhas, as brotações que emergem das cepas remanescentes após a colheita da madeira de eucalipto também apresentam grande potencial competitivo, necessitando de métodos de controle que levam ao uso excessivo de herbicidas devido à elevada taxa de crescimento inicial do eucalipto e sua capacidade de exploração dos recursos de crescimento (MACHADO et al., 2010).

A capacidade de emissão de brotações nas espécies do gênero Eucalyptus está associada à presença de gemas adventícias, brotos epicórmicos e lignotubers na casca e na região cambial (CARR; JAHNKE; CARR, 1984; CLARKE et al., 2013; FERRAZ FILHO; SCOLFORO; MOLA-YUDEGO, 2014). A emissão de novas brotações geralmente é induzida após distúrbios como incêndios (CLARKE et al., 2013) ou pelo próprio corte das árvores, após atividades de colheita florestal. Apesar das vantagens relacionadas à capacidade de rebrota do eucalipto, principalmente em relação à possibilidade de condução de uma segunda rotação pelo sistema de talhadia (FERRAZ FILHO; SCOLFORO; MOLA-YUDEGO, 2014), torna-se necessário o controle das brotações, a fim de evitar efeitos competitivos com as novas mudas plantadas ou com o broto selecionado para condução.

Entre as formas de manejo de brotações destacam-se os métodos de controle manual e químico. De maneira geral, o método de controle químico apresenta baixo custo operacional por área, maior velocidade de operação e maior eficiência de controle (MACHADO et al., 2010). O manejo químico convencional é realizado a partir da pulverização de glyphosate sobre brotações com porte de altura entre 0,90 e 1,20 m. Porém, muitas vezes, mesmo com a utilização do manejo químico, observam-se baixos níveis de controle de brotações, o que leva a realizar novas aplicações em uma mesma área. Em seus estudos, Roberts, Little e Light (2016) observaram que foram necessárias pelo menos quatro aplicações para atingir nível satisfatório de controle de brotações de Eucalyptus urophylla x Eucalyptus grandis, com gasto total de 
glyphosate (360 g i.a. $\mathrm{L}^{-1}$ ) variando de aproximadamente 13 a $20 \mathrm{~L} \mathrm{ha}^{-1}$. Em casos como este, quando o manejo químico apresenta baixa eficiência de controle, o método de controle manual pode ser realizado de forma complementar (MEDAUAR et al.., 2018), porém, tal método também apresenta reduzidos níveis de controle (LITTLE, 2003; ROBERTS; LITTLE; LIGHT, 2016) devido ao elevado vigor das brotações e a má utilização das tecnologias de aplicação.

Outra forma de manejo químico de brotações de eucalipto é a aplicação de herbicidas diretamente no fuste antes do corte raso (LITTLE, 2003; ROBERTS; LITTLE; LIGHT, 2017), entretanto, poucos trabalhos relatam a eficiência de controle de brotações de eucalipto com este método de aplicação. Apesar de ser uma aplicação direcionada, mais segura para o ambiente, sabe-se que esse método de aplicação é pouco difundido em empresas florestais situadas no Brasil e que não há relatos na literatura sobre os níveis de eficiência de controle nas condições edafoclimáticas do país.

Considerando o fato de que o controle de brotações indesejadas de eucalipto constitui um grande desafio para silvicultura, sobretudo pelo alto número de operações e gasto excessivo de produtos (LITTLE, 2003, ROBERTS; LITTLE; LIGHT, 2016; MEDAUAR et al., 2018), levantou-se a hipótese de que a aplicação localizada dos herbicidas no fuste principal das árvores do eucalipto, em fase pré-corte raso, pode potencializar o seu controle de brotações ou mesmo reduzir seu crescimento e vigor. Assim, estudos relacionados à adoção desse método de aplicação nas condições edafoclimáticas do país são importantes para ampliar a efetividade das atividades de controle de brotações de eucalipto de forma segura e com menor risco ao ambiente. Por isso, neste trabalho objetivou-se avaliar a eficiência dos herbicidas glyphosate e imazapyr, aplicados de forma localizada em inserções no fuste principal das árvores, na fase précorte, para controle de brotações de eucalipto. 


\section{MATERIAL E MÉTODOS}

O experimento foi conduzido no município de Itacambira - MG, na empresa GELF Siderurgia S/A, entre os meses de junho a dezembro de 2018. O clima da região é classificado como Cwb, subtropical úmido com inverno seco e verão temperado (ALVARES et al., 2013), com altitude de aproximadamente 1217 metros. Os dados referentes à precipitação mensal $(\mathrm{mm})$ e temperatura média mensal $\left({ }^{\circ} \mathrm{C}\right)$ durante $\mathrm{O}$ período de implantação, condução e avaliação dos experimentos estão representados na Figura 1.

Figura 1 - Precipitação média mensal $(\mathrm{mm})$ e temperatura média mensal $\left({ }^{\circ} \mathrm{C}\right)$ na área experimental, no município de Itacambira - MG, durante o período de junho a dezembro de 2018

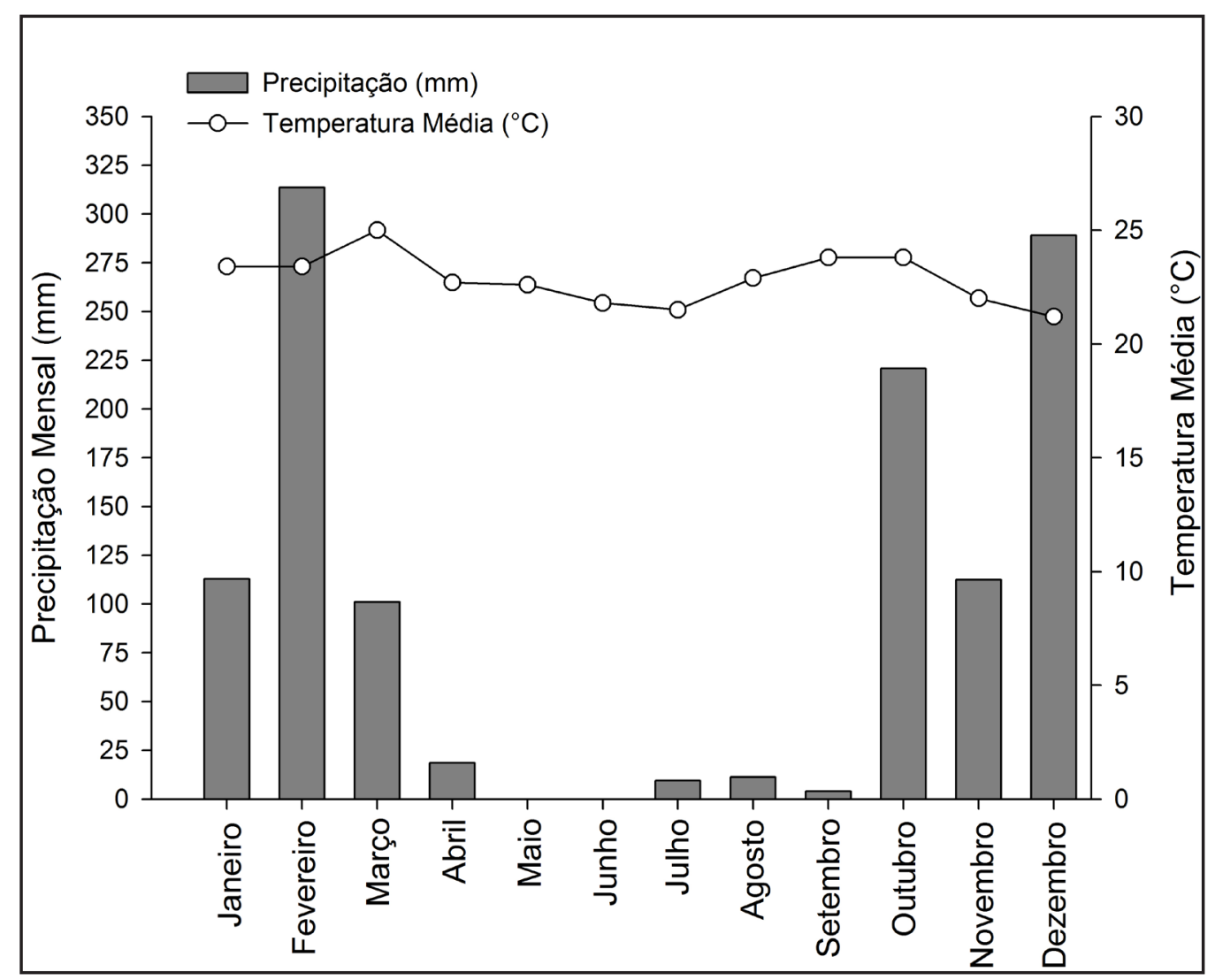

Fonte: Autores (2019) 
Aárea experimental correspondeu a um talhão conduzido em sistema de talhadia de um clone Eucalyptus grandis x Eucalyptus urophylla plantado no espaçamento de $3 \times$ 3 m. O plantio inicial foi realizado em 2005 e conduzido até 2012, quando foi realizado o primeiro corte do fuste útil. Em 2012, iniciou-se o manejo da área para condução das brotações pelo sistema de talhadia.

O experimento foi implantado em delineamento em blocos casualizados com quatro repetições. Foram implantados sete tratamentos correspondentes a doses de glyphosate e imazapyr aplicados em pré-corte (Tabela 1). Para a aplicação de glyphosate e imazapyr foram utilizados os produtos comerciais Gli-Up ${ }^{\circledR}$ (360 g i.a. L-1) e Chopper Florestal ${ }^{\circledR}$ (250 g e.a. L-1 $^{-1}$, respectivamente. Cada parcela experimental foi representada por 10 árvores de eucalipto.

Tabela 1 - Descrição dos tratamentos, com aplicação de doses de glyphosate (Gli-Up ${ }^{\circledR}$ - 360 g i.a. $\mathrm{L}^{-1}$ ) e imazapyr (Chopper Florestal ${ }^{\circledR}$ - 250 g e.a. $\mathrm{L}^{-1}$ ) aplicados em pré-corte sobre inserções realizadas no fuste de árvores eucalipto

\begin{tabular}{|c|c|c|c|}
\hline Tratamento & $\begin{array}{c}\text { Dose } \\
\text { (mL herbicida/fuste) }\end{array}$ & $\begin{array}{c}\text { Dose } \\
(\text { L ha-1)* }\end{array}$ & $\begin{array}{c}\text { Dose do princípio } \\
\text { ativo (g i.a. ha-1) }\end{array}$ \\
\hline Testemunha & - & - & - \\
\hline Imazapyr & 0,3 & 0,333 & 0,083 \\
\hline Imazapyr & 0,5 & 0,556 & 0,139 \\
\hline Imazapyr & 0,7 & 0,778 & 0,195 \\
\hline Glyphosate & 3,0 & 3,333 & 1,200 \\
\hline Glyphosate & 4,0 & 4,444 & 1,600 \\
\hline Glyphosate & 5,0 & 5,555 & 2,000 \\
\hline
\end{tabular}

Fonte: Autores (2019)

Em que: * Considerando 1111 árvores por hectare (espaçamento de $3 \times 3$ m).

Os herbicidas, em suas respectivas doses, foram aplicados diretamente no fuste das árvores a partir de uma inserção realizada com "machadinha" a aproximadamente 1,30 m de altura, com profundidade de 3 a 4 centímetros. A aplicação foi realizada com auxílio de seringas dosadoras de fluxo contínuo, que possibilitam dosagens de 0,1 a 5,0 mL. A aplicação dos herbicidas foi realizada em 26 de junho de 2018 e o corte das árvores foi realizado aos 30 dias após a aplicação dos herbicidas, com 
auxílio de motosserra. Após o corte, os fustes úteis das árvores foram retirados da área experimental e empilhados na borda do talhão.

Após o corte das árvores, realizou-se o acompanhamento da emissão das brotações nas cepas dos tratamentos-controle e nas cepas adjacentes à área experimental para determinar o início do período das avaliações. Assim, as avaliações foram iniciadas aos 150 dias após corte das árvores. Dessa forma, foram realizadas mensurações de altura, do número de brotações emergidas por cepa e da porcentagem de cepas com presença de brotações nas 10 cepas de cada parcela experimental. Adicionalmente, para avaliar a eficiência de controle das brotações indesejadas, foi elaborada uma escala de eficiência de controle (Figura 2). A escala foi elaborada considerando-se o porte, vigor e capacidade de desenvolvimento das brotações. A avaliação de eficiência de controle consistiu da atribuição de notas de 0 a $100 \%$, em que 0 correspondeu à ausência de controle e 100\% correspondeu ao controle total das brotações.

Figura 2 - Escala de eficiência de controle de brotações de um clone Eucalyptus grandis x Eucalyptus urophylla aos 150 dias após aplicação em pré-corte de doses de glyphosate e imazapyr

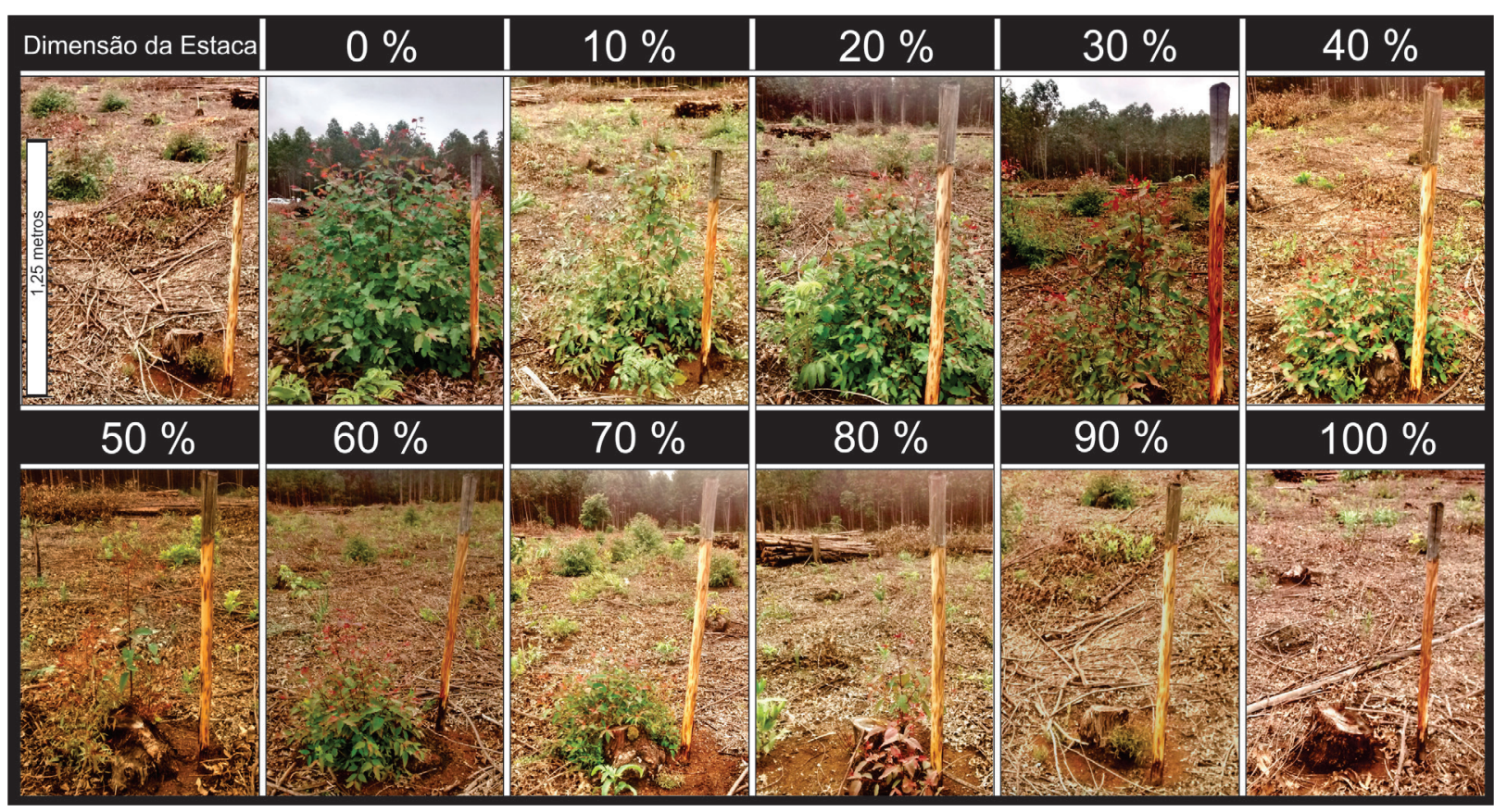

Fonte: Autores (2019) 
Os dados obtidos foram submetidos à análise de normalidade dos resíduos pelo teste Shapiro-Wilk e à análise de homogeneidade de variâncias pelo teste de Bartlett. As variáveis que atenderam aos pressupostos anteriores foram submetidas à análise de variância pelo teste $F(p \leq 0,05)$ e realizou-se comparação das médias entre os tratamentos pelo teste Tukey $(p \leq 0,05)$. Demais variáveis foram analisadas de forma descritiva em função do erro padrão da média. Todas as análises foram realizadas com auxílio do software RStudio ${ }^{\circledR}$ (R DEVELOPMENT CORE TEAM, 2020).

\section{RESULTADOS E DISCUSSÃO}

A aplicação do herbicida glyphosate sobre o fuste de árvores de Eucalyptus grandis x Eucalyptus urophylla, no pré-corte raso do povoamento, apresentou porcentagens de controles promissoras, o que não foi verificado com a aplicação do imazapyr. A aplicação de doses de herbicida propiciou efeito significativo $(p \leq 0,05)$ em todas as características avaliadas. No entanto, após a aplicação de doses de imazapyr, verificou-se o surgimento médio de três ou mais brotações por cepa $(p \leq 0,05)$ e a emissão de brotações em pelo menos $97,5 \%$ das cepas, médias semelhantes às encontradas no tratamento testemunha (Figura 3). A aplicação das doses de 3,0 e 4,0 $\mathrm{mL}$ de glyphosate promoveu redução dos valores médios do número de brotações emitidos por cepa quando comparado às cepas provenientes de árvores que não foram submetidas à aplicação de herbicida $(p \leq 0,05)$. Porém, observou-se que apenas com a aplicação da dose de $3 \mathrm{~mL}$ por árvore de glyphosate houve leve redução dos valores médios de presença de brotações, apesar de ainda assim ter sido observada a emissão de brotações em aproximadamente $80 \%$ das cepas (Figura 3). Nas condições experimentais do presente estudo, verificou-se que seria necessária uma nova intervenção de controle para efetiva eliminação das brotações de eucalipto. 
Figura 3 - Percentual médio de cepas com emissão de brotações (a) e número de brotações por cepa (b) em função de doses de glyphosate (Gli-Up ${ }^{\circledR} 360$ g i.a. L L'-1) e imazapyr (Chopper Florestal ${ }^{\circledR} 250$ g i.a. L-1) aplicadas de forma dirigida em pré-corte sobre o fuste de um clone Eucalyptus grandis $\mathrm{x}$ Eucalyptus urophylla

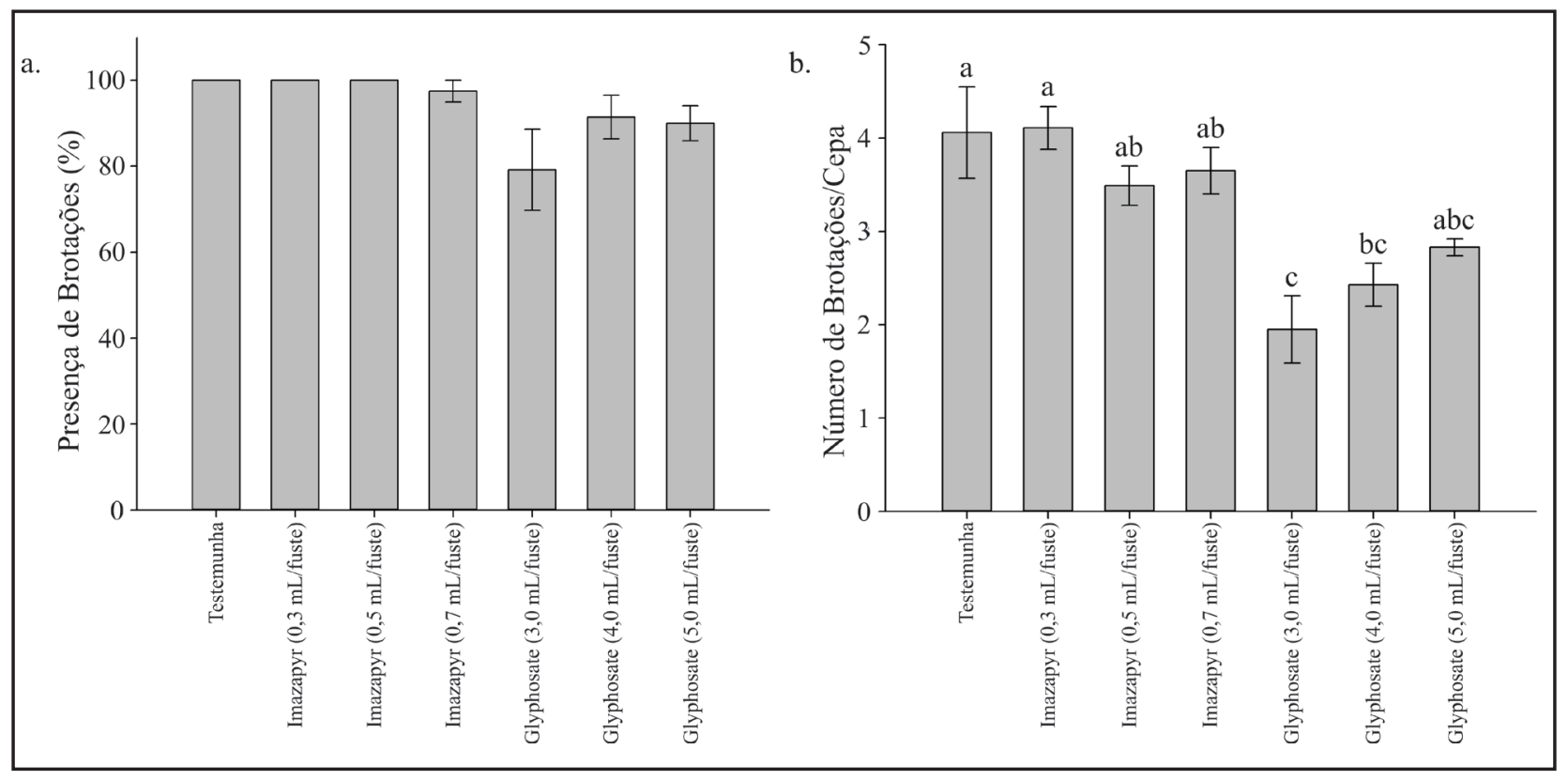

Fonte: Autores (2019)

Em que: *Médias seguidas por letras iguais nas colunas não diferem significativamente entre si (Tukey $\mathrm{p} \leq 0,05)$. Barras = erro padrão da média.

As cepas provenientes de árvores que não foram submetidas à aplicação de herbicidas apresentaram 100\% de emissão de brotações, com médias de altura e número de brotações por cepa de 1,23 m e 4,06, respectivamente (Figuras 3 e 4). Tais valores justificam a necessidade de controle das brotações antes do período de reforma, devido à elevada capacidade de emissão e vigor dessas brotações.

A aplicação de doses de imazapyr propiciou eficiência de controle inferior a 25\% (Figura 4), demostrando o reduzido potencial da utilização de imazapyr aplicado no fuste das árvores em pré-corte para controle de brotações. A aplicação de doses de glyphosate foi mais eficiente que o imazapyr no controle de brotações, com redução da altura e promoção de maiores valores de eficiência de controle. A aplicação de $3 \mathrm{~mL}$ de glyphosate promoveu, aos 150 dias após corte das árvores, altura média de brotações 
igual a 0,23 m e eficiência de controle de $81 \%$ (Figura 4). Dessa forma, a redução no vigor e desenvolvimento das brotações de eucalipto a partir da aplicação direta de glyphosate no fuste principal das árvores no pré-corte pode tornar as brotações mais susceptíveis a uma atividade adicional de controle e auxiliar na sua efetiva eliminação.

Figura 4 - Eficiência de controle (a) e altura média (b) de brotações em função de doses de glyphosate (Gli-Up ${ }^{\circledR} 360$ g i.a. L $\left.^{-1}\right)$ e imazapyr (Chopper Florestal ${ }^{\circledR} 250$ g i.a. L $^{-1}$ ) aplicadas de forma dirigida em pré-corte sobre o fuste de um clone Eucalyptus grandis $\times$ Eucalyptus urophylla

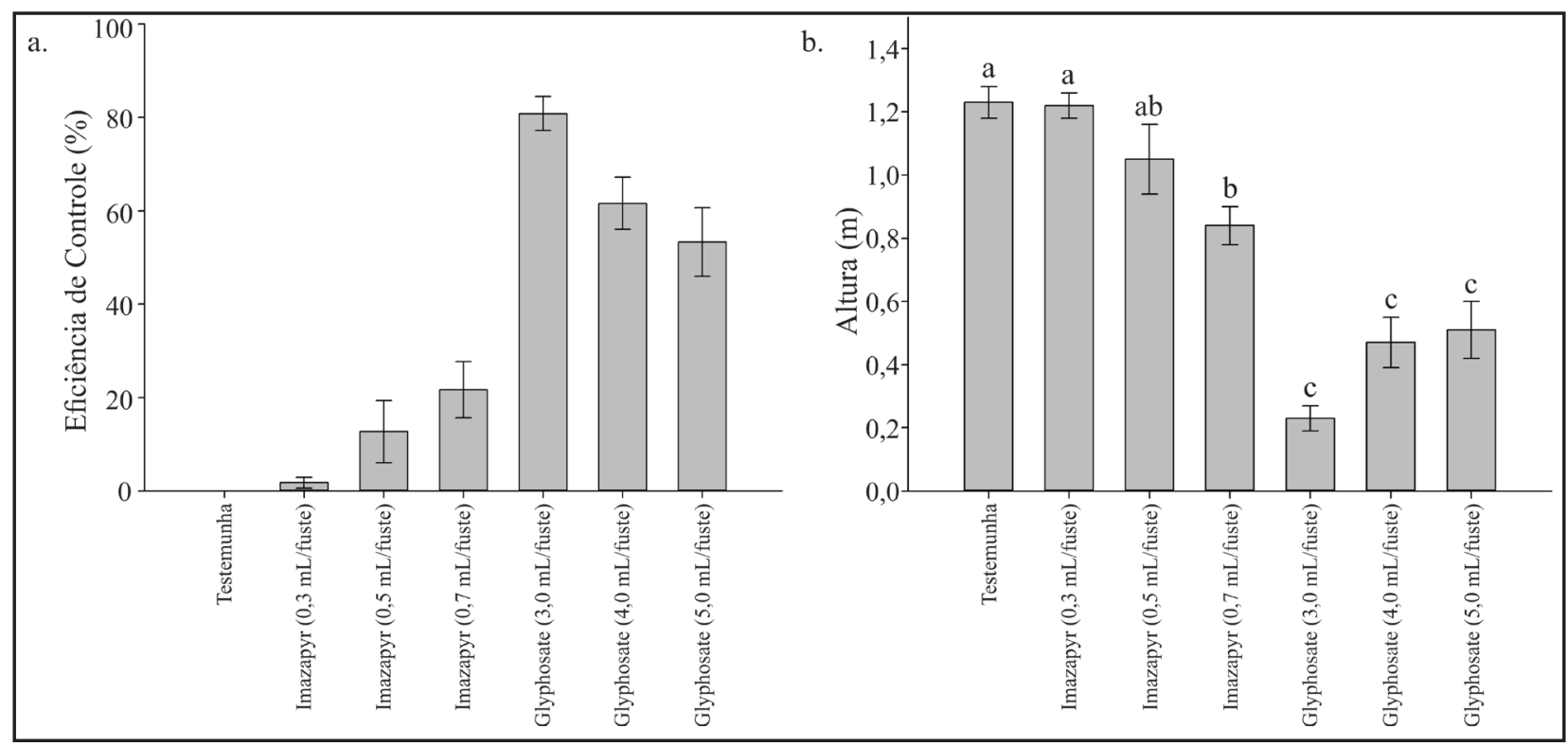

Fonte: Autores (2019)

Em que: *Médias seguidas por letras iguais nas colunas não diferem significativamente entre si (Tukey $p \leq 0,05)$. Barras = erro padrão da média.

Variações nos índices de controle de brotações a partir de aplicações de herbicida com aplicação no pré-corte raso das árvores são conhecidas. Little (2003), ao comparar métodos de controle de brotações de Eucalyptus grandis, observou que a aplicação em pré-corte das árvores apresentou melhores resultados em relação à aplicação sobre a superfície das cepas após corte ou pulverização sobre as brotações. Adicionalmente, Roberts, Little e Light (2017) observaram que a aplicação de triclopyr de forma dirigida no fuste um dia antes do corte de árvores de Eucalyptus macarthurii promoveu controle 
de $87 \%$ das cepas, aproximadamente, 98 dias após aplicação. Porém, esses mesmos autores observaram que foram necessárias operações adicionais para o controle total das brotações.

Tais diferenças em relação ao controle podem ser relacionadas às variações entre espécies, conforme observado por Tuffi Santos et al. $(2006 ; 2007)$ que verificaram diferenças na tolerância de diferentes espécies de eucalipto ao glyphosate. Os herbicidas quando aplicados necessitam de eficiente absorção e translocação para promoverem controle das brotações. Em pulverizações convencionais, os herbicidas são aplicados sobre a biomassa aérea das brotações, absorvidos pelas folhas e translocados para diferentes partes da planta. Entretanto, no método de aplicação pré-corte, a aplicação é realizada de forma dirigida no fuste das árvores antes do corte raso. Assim, os herbicidas aplicados de forma localizada em inserção no fuste das árvores atingem diretamente os vasos do xilema e floema, nos quais são translocados para os pontos de crescimento das plantas.

As diferenças de resposta de controle das brotações de eucalipto em função das formas de aplicação e de doses de herbicidas também podem ser atribuídas às condições ambientais locais. As espécies do gênero Eucalyptus podem apresentar respostas distintas relacionadas à capacidade de adaptação a condições ambientais, como por exemplo, de estresse hídrico (CORREIA et al., 2014; BOURNE; HAIGH; ELLSWORTH, 2015; HODECKER et al., 2018). Em períodos ou ambientes em que há limitação hídrica, as plantas apresentam redução do desenvolvimento foliar e promovem o fechamento estomático que leva à redução nas trocas gasosas e, consequentemente, na atividade fotossintética (SHAO et al., 2008; BOURNE; HAIGH; ELLSWORTH, 2015; GONÇALVES et al., 2017; TAIZ et al., 2017). Nesses casos, a limitação de trocas gasosas ocasionada pelo fechamento estomático pode influenciar direta e indiretamente a translocação de solutos nas plantas (TAlZ et al., 2017), assim como a translocação de herbicidas, já que tanto o glyphosate quanto o imazapyr apresentam translocação preferencial pelo floema (SILVA; FERREIRA; FERREIRA, 2007). Dessa forma, variações ambientais 
que alterem a capacidade de translocação da seiva e atividade fisiológica das plantas podem afetar a translocação dos herbicidas e, consequentemente, a eficiência do controle das brotações. Dye et al. (2016) observaram redução no fluxo de seiva de duas espécies de eucalipto no período de inverno, coincidente com períodos de baixa precipitação pluviométrica. Adicionalmente, os mesmos autores observaram que houve aumento das taxas de fluxo de seiva a partir do início do período chuvoso. De forma semelhante, Pinto Junior et al. (2013) também observaram redução do fluxo de seiva no período seco e posterior aumento do fluxo de seiva no período úmido em quatro espécies arbóreas da floresta de transição Amazônia Cerrado. Neste trabalho, a aplicação dos herbicidas foi realizada no período de inverno, período em que houve redução nos índices de precipitação (Figura 1). Assim, os valores de eficiência de controle obtidos podem ter sido influenciados negativamente por variações na translocação dos herbicidas, devido ao período de baixos índices pluviométricos.

A decisão final para escolha de determinado método de controle de brotações deve basear-se nos custos operacionais concomitantemente com a escolha de um método em que o controle atinja níveis satisfatórios com apenas uma aplicação sem riscos às mudas implantadas posteriormente (LITTLE, 2003). Apesar de ter sido observada redução do vigor das brotações a partir da aplicação de glyphosate, ressaltase que com os valores obtidos tanto de presença de brotações quanto de eficiência de controle seriam necessárias intervenções de controle adicionais para atingir níveis satisfatórios de eliminação das brotações.

Embora o método de controle pré-corte apresente demanda de novas intervenções de controle, esse método pode vir a favorecer a redução do número de operações necessárias após o corte das árvores para controle de brotações. Roberts, Little e Light (2017) observaram a necessidade de uma operação de pulverização, além de quatro operações manuais para controle de brotações de Eucalyptus macarthurii. Adicionalmente, Roberts, Little e Light (2016), ao avaliarem o controle de brotações de Eucalyptus grandis x Eucalyptus urophylla com porte de altura de 0,5; 1,0 ou 1,50 m a partir da aplicação de diferentes concentrações de glyphosate, observaram a 
necessidade de pelo menos quatro intervenções de controle, independentemente do porte das brotações ou concentração de glyphosate. Dessa forma, como o método de controle pré-corte apresentou, a partir da aplicação de glyphosate, redução no vigor das brotações, esse método pode ser utilizado de forma complementar, com o objetivo de reduzir o número de pulverizações realizadas com o método de controle convencional.

A busca por práticas que aperfeiçoem as atividades de aplicação de herbicida são demandas do setor florestal (TUFFI SANTOS et al., 2008). Durante operações convencionais de pulverização, há possibilidade de ocorrência de deriva da calda herbicida que pode atingir e afetar o desenvolvimento de plantas não alvo. Nesse caso, o contato direto do glyphosate em plantas de eucalipto pode promover sintomas de intoxicação e alteração no crescimento das plantas (SANTOS JÚNIOR et al., 2015; SALGADO et al., 2017; SANTOS et al., 2019). Na aplicação localizada de herbicidas em inserção direta no fuste das árvores de eucalipto antes do corte raso, a ocorrência de deriva e o contato direto do herbicida com organismos não alvo, solo ou corpos hídricos é praticamente nula. Com isso, ainda que os índices de controle não tenham sido altamente eficientes neste trabalho, buscas por melhorias relacionadas à eficiência de controle a partir dessa forma de aplicação devem ser mantidas, com intuito de auxiliar no desenvolvimento das atividades operacionais da silvicultura, com mitigação de problemas ambientais.

\section{CONCLUSÕES}

A aplicação de glyphosate no fuste das árvores em pré-corte promove redução no número e desenvolvimento das brotações indesejadas de eucalipto, porém com níveis medianos de controle, sendo necessárias operações adicionais de manejo para atingir níveis satisfatórios de controle. A aplicação de imazapyr localizada em inserção no fuste das árvores em pré-corte, nas doses testadas, é ineficiente no controle de brotações de eucalipto. 


\section{AGRADECIMENTOS}

Os autores agradecem à Fundação de Amparo à Pesquisa do Estado de Minas Gerais (FAPEMIG), ao Conselho Nacional de Desenvolvimento Científico e Tecnológico (CNPq) e à Coordenação de Aperfeiçoamento de Pessoal de Nível Superior (CAPES) pelo auxílio financeiro. Adicionalmente, também agradecem a GELF Siderurgia S/A pela disponibilidade da área experimental e suporte para desenvolvimento dos experimentos.

\section{REFERÊNCIAS}

ALVARES, C. A. et al. Köppen's climate classification map for Brazil. Meteorologische Zeitschrift, Berlin, v. 22, n. 6, p. 711-728, dec. 2013.

BAUHUS, J.; VAN DER MEER, P. J.; KANNINEN, M. Ecosystem Goods and Services from Plantation Forests. [S. I.]: Earthscan, 2010. 276 p.

BOURNE, A. E.; HAIGH, A. M.; ELLSWORTH, D. S. Stomatal sensitivity to vapour pressure deficit relates to climate of origin in Eucalyptus species. Tree Physiology, Oxford, v. 35, n. 3, p. 266278, mar. 2015.

CARR, D.; JAHNKE, R.; CARR, S. Initiation, development and anatomy of lignotubers in some species of eucalyptus. Australian Journal of Botany, Melbourne, v. 32, n. 4, p. 415-437, 1984.

CLARKE, P. J. et al. Resprouting as a key functional trait: how buds, protection and resources drive persistence after fire. New Phytologist, Cambridge, v. 197, n. 1, p. 19-35, jan. 2013.

CORREIA, B. et al. Water stress and recovery in the performance of two Eucalyptus globulus clones: physiological and biochemical profiles. Physiologia Plantarum, Copenhagen, v. 150, n. 4, p. 580-592, abr. 2014.

DYE, P. et al. The annual pattern of sap flow in two Eucalyptus species established in the vicinity of gold-mine tailings dams in central South Africa. Southern Forests: a Journal of Forest Science, South Africa, v. 78, n. 4, p. 307-313, aug. 2016.

FERRAZ FILHO, A. C.; SCOLFORO, J. R. S.; MOLA-YUDEGO, B. The coppice-with-standards silvicultural system as applied to Eucalyptus plantations - a review. Journal of Forestry Research, [s. I.], v. 25, n. 2, p. 237-248, abr. 2014.

GONÇALVES, J. L. et al. Eucalypt plantation management in regions with water stress. Southern Forests: a Journal of Forest Science, South Africa, v. 79, n. 3; p. 169-183, may 2017.

HODECKER, B. E. R. et al. Water availability preceding long-term drought defines the tolerance of Eucalyptus to water restriction. New Forests, Dordrecht, v. 49, n. 2, p. 173-195, out. 2018. 
LITTLE, K. M. Killing Eucalyptus grandis cut stumps after multiple coppice rotations in the KwaZulu-Natal midlands, South Africa. The Southern African Forestry Journal, South Africa, v. 199, n. 1, p. 7-13, 2003.

MACHADO, A. F. L. et al. Interferência de plantas daninhas na cultura do eucalipto. In: FERREIRA, L. R. et al. (org.). Manejo integrado de plantas daninhas na cultura do eucalipto. 1. ed. Viçosa, MG: Editora UFV, 2010. v. 1. p. 15-37.

MEDAUAR, C. C. et al. Espectro de gotas e distribuição de calda herbicida associada a fertilizante foliar em áreas de reforma florestal. Scientia Forestalis, Piracicaba, v. 46, n. 119, p. 333-345, set. 2018.

PAYN, T. et al. Changes in planted forests and future global implications. Forest Ecology and Management, Amsterdam, v. 352, n. 7, p. 57-67, set. 2015.

PINTOJUNIOR, O. B. et al. Transpiração pelo método da sonda de dissipação térmica em floresta de transição amazônica-cerrado. Revista Brasileira de Engenharia Agrícola e Ambiental, Campina Grande, v. 17, n. 3, p. 268-274, 2013.

R DEVELOPMENT CORE TEAM. R: a Language and Environment for Statistical Computing. Vienna: R Foundation for Statistical Computing, 2020.

ROBERTS, J. C.; LITTLE, K. M.; LIGHT, M. E. A comparison of the cost-effectiveness of different eucalypt cut-stump control management options to reduce competition from coppice regrowth during stand establishment in Mpumalanga, South Africa. Southern Forests: a Journal of Forest Science, South Africa, v. 80, n. 3, p. 261-268, dec. 2017.

ROBERTS, J. C.; LITTLE, K. M.; LIGHT, M. E. The use of glyphosate for the management of secondary coppice regrowth in a Eucalyptus grandis $\times E$. urophylla coppice stand in Zululand, South Africa. Southern Forests: a Journal of Forest Science, South Africa, v. 78, n. 3, p. 217223, may 2016.

SALGADO, T. P. et al. Effects of glyphosate on growth, yield and wood of Eucalyptus grandis. Journal of Tropical Forest Science, Kuala Lumpur, v. 29, n. 3, p. 257-266, jul. 2017.

SANTOS JUNIOR, A. et al. Glyphosate drift in eucalyptus plants. Planta Daninha, Viçosa, MG, v. 33, n. 3, p. 615-621, set. 2015.

SANTOS, S. A. et al. Carfentrazone-ethyl and glyphosate drift inhibits uredinial formation of Austropuccinia psidii on Eucalyptus grandis leaves. Pest Management Science, Sussex, v. 75, n. 1, p. 53-62, jan. 2019.

$\mathrm{SHAO}, \mathrm{H}$. B. et al. Water-deficit stress-induced anatomical changes in higher plants. Comptes Rendus Biologies, Paris, v. 331, n. 3, p. 215-225, jan. 2008.

SILVA, A. A.; FERREIRA, F. A.; FERREIRA, L. R. Herbicidas: Classificação e Mecanismos de Ação. In: SILVA, A. S., SILVA, A. A. Tópicos em manejo de plantas daninhas. Viçosa, MG: UFV, 2007. p. 58-117. 
TAIZ, L. et al. Fisiologia e desenvolvimento vegetal. 6. ed. Porto Alegre: Artmed, 2017.

TUFFI SANTOS, L. D. et al. Danos visuais e anatômicos causados pelo glyphosate em folhas de Eucalyptus grandis. Planta Daninha, Viçosa, MG, v. 26, n. 1, p. 9-16, jan. 2008.

TUFFI SANTOS, L. D. et al. Intoxicação de espécies de eucalipto submetidas à deriva do glyphosate. Planta Daninha, Viçosa, MG, v. 24, n. 2, p. 359-364, 2006.

TUFFI SANTOS, L. D. et al. Morphological responses of different eucalypt clones submitted to glyphosate drift. Environmental and Experimental Botany, Elmsford, v. 59, n. 1, p. 11-20, 2007.

VIDAL, J. M. et al. Preservação de madeiras no brasil: histórico, cenário atual e tendências. Ciência Florestal, Santa Maria, v. 25, n. 1, p. 257-271, jan. 2015.

VILLAR, E. et al. RNA-Seq reveals genotype-specific molecular responses to water deficit in eucalyptus. BMC Genomics, [s. I.], v. 12, n. 1, p. 1-18, nov. 2011.

\section{Contribuição de Autoria}

\section{1 - William Gomes Montes}

Engenheiro Florestal, Me., Analista Florestal

https://orcid.org/0000-0001-9467-8741•william.florestal@outlook.com

Contribuição: Administração do projeto, Conceituação, Curadoria de dados, Análise Formal, Visualização de dados, Investigação, Metodologia, Escrita - primeira redação, Escrita - revisão e edição

\section{2 - Leonardo David Tuffi Santos}

Agrônomo, Dr., Professor

https://orcid.org/0000-0002-9362-778X•Ituffi@ica.ufmg.br

Contribuição: Administração do projeto, Obtenção de financiamento, Conceituação, Análise Formal, Visualização de dados, Metodologia, Escrita - primeira redação, Escrita - revisão e edição

\section{3 - Rodrigo Eduardo Barros}

Agrônomo, Me., Doutorando em Produção Vegetal https://orcid.org/0000-0003-4785-7725•rodrigo.edb@hotmail.com

Contribuição: Conceituação, Curadoria de dados, Análise Formal, Visualização de dados, Investigação, Supervisão, Metodologia, Escrita - revisão e edição 


\section{4 - Wellington Almeida}

Engenheiro Florestal, Gerente Técnico

https://orcid.org/0000-0002-1259-3961•wellington.almeida@gelfsid.com.br

Contribuição: Conceituação, Análise Formal, Visualização de dados, Obtenção de Financiamento, Escrita - revisão e edição

\section{5 - Antônio Junior Silva Abreu}

Engenheiro de Produção, Supervisor Técnico

https://orcid.org/0000-0002-1696-2023•antonio.abreu@gelfsid.com.br

Contribuição: Conceituação, Análise Formal, Visualização de dados, Recursos, Escrita - revisão e edição

\section{6 - Matheus Mendes Reis}

Engenheiro Agrícola e Ambiental, Dr., Professor

https://orcid.org/0000-0003-2100-2438•matheussmendes@hotmail.com

Contribuição: Curadoria de dados, Análise Formal, Visualização de dados, Metodologia, Validação, Software, Escrita - revisão e edição

\section{7 - Luan Mateus Silva Donato}

Agrônomo, Dr., Técnico em Agropecuária

https://orcid.org/0000-0002-3906-2431 • luan_mateus_sd@hotmail.com

Contribuição: Curadoria de dados, Análise Formal, Visualização de dados, Metodologia, Validação, Software, Escrita - revisão e edição

\section{8 - Guilherme Augusto de Paiva Ferreira}

Agrônomo, Mestrando em Produção Vegetal

https://orcid.org/0000-0001-6266-9963•guilhermepaiva017@gmail.com

Contribuição: Curadoria de dados, Análise Formal, Visualização de dados, Metodologia, Validação, Escrita - revisão e edição

\section{Como citar este artigo}

Montes, W. G.; Santos, L. D. F.; Barros, R. E.; Almeida, W.; Abreu, A. J. S.; Reis, M. M.; Donato, L. M. S.; Ferreira, G. A. P. Glyphosate e imazapyr no controle de brotações de eucalipto: aplicação no fuste principal anterior ao corte da árvore. Ciência Florestal, Santa Maria, v. 31, n. 4, p. 1654-1670, 2021. DOI 10.5902/1980509840102. Disponível em: https://doi.org/10.5902/1980509840102. 